\title{
Scaling through ocean carbon
}

\author{
Marine microbes have shaped the climate throughout Earth's history. Integration of microbial carbon cycling \\ dynamics across a range of spatial scales will be critical for understanding the ocean's impact in light of a \\ changing climate.
}

$\mathrm{n}$ $\mathrm{n}$ the surface ocean, photosynthetic microbes fix inorganic carbon - some of which comes from exchange with $\mathrm{CO}_{2}$ in the atmosphere - into organic matter, most of which is recycled back into the water column by other microbes. A fraction of the organic matter, though, sinks to the deep ocean as particulate organic matter. Carbon can be locked away from returning to the atmosphere via this biological carbon pump for thousands of years or longer ${ }^{1}$. The amount of sequestered carbon is determined by rates of photosynthetic carbon fixation in the surface ocean, the speed at which resulting particles sink, and how quickly microbial respiration can metabolize particulate organic matter back to inorganic carbon - also called remineralization before it's locked away in the seabed. There are still many unknowns about the mechanisms that govern ocean carbon cycling. In this issue, two Articles each tackle knowledge gaps in understanding the carbon cycle across temporal and spatial scales. Together they emphasize the critical need to integrate the microscale with the macroscale to predict the fate of carbon in a rapidly changing ocean.

The past can offer hints about how climate shapes the ocean carbon cycle. The complex interactions of the biological carbon pump mean that the flux of carbon from the atmosphere to the ocean's interior has not remained constant over geological time. In the 15 million years since the middle Miocene, as global surface ocean temperatures gradually dropped by about $5{ }^{\circ} \mathrm{C}$, rates of microbial organic matter metabolism by microbes also declined. Sluggish metabolic breakdown means more carbon evades consumption and sinks to the deep ocean. The efficiency of the biological carbon pump increased in lockstep with ocean cooling over the past 15 million years ${ }^{2}$, lowering $\mathrm{CO}_{2}$ in the atmosphere and preventing additional warming. In contrast, in modern times anthropogenic greenhouse gas emissions are heating the surface ocean ${ }^{3}$. Warmer water could shift the gears of the biological carbon pump in different ways with speedier microbial metabolic rates and elevated dissolved $\mathrm{CO}_{2}$ production. Some of that $\mathrm{CO}_{2}$ will make it back into the atmosphere, potentially leading to a positive feedback on global warming, however the mechanisms and consequences of an altered ocean carbon cycle remain largely unknown.

\section{"Integrate the microscale with the macroscale to predict the fate of carbon in a rapidly changing ocean."}

On the microscale, particle sinking speed and the rate of microbial organic matter metabolism are in a race against each other. On slowly sinking particles, microbes can break down more organic matter, churning out inorganic carbon including dissolved $\mathrm{CO}_{2}$ nearer the surface where it can be returned to the atmosphere. Fast-sinking particles deliver organic matter to the seabed before microbes can break it all down. Conventional thinking held that sinking and degradation rates were independent variables in the control of the biological carbon pump. However, in controlled experiments that varied the flow rate around particles, in an Article in this issue, Alcolombri et al. find that the opposite is true; the faster a particle sinks, the higher the rate of bacterial degradation. Fast-sinking particles do not necessarily equate to a more efficient biological carbon pump. When considering the climate impacts of larger-scale ocean processes, these particle-level interactions might have a big effect on where carbon ends up.

At a larger spatial scale, in an Article, Johnson et al. reconstruct the frequency of phytoplankton blooms in the Southern Ocean around Antarctica, a key region for global carbon cycling driven by high rates of primary productivity. Using sediment records, they find the frequency of bloom events sizably decreased 4,500 years ago. This shift, triggered by increases in sea ice extent due to El Niño-Southern Oscillation teleconnections, likely decreased primary productivity and the amount of carbon sequestered away from the atmosphere. Regional climate-carbon cycle connections could have wide-spread impacts, as feedbacks drive further warming at the planetary scale.

The ocean carbon cycle can be investigated across a range of spatiotemporal scales. As knowledge gaps in the ocean carbon cycle are filled from particle- to planetary-levels, one clear picture that emerges is the mutability of climatically important processes like the biological carbon pump. Moving forward into a world with a climate markedly different than the recent past, the challenge will be to expand research that successfully integrates the micro with the macro.

Published online: 4 October 2021 https://doi.org/10.1038/s41561-021-00840-y

\footnotetext{
References

1. Jiao, N. et al. Nat. Rev. Microbiol. 8, 593-599 (2010).

2. Boscolo-Galazzo, F. et al. Science 371, 1148-1152 (2021).

3. Gleckler, P. et al. Nat. Clim. Change 2, 524-529 (2012).
} 